\title{
The magnetic precursor of L1448-mm: excitation differences between ion and neutral fluids
}

\author{
J. F. Roberts ${ }^{1,2}$, I. Jiménez-Serra ${ }^{3,4}$, J. Martín-Pintado ${ }^{1}$, S. Viti ${ }^{2}$, A. Rodríguez-Franco ${ }^{1,5}$, A. Faure ${ }^{6}$, and J. Tennyson ${ }^{2}$ \\ ${ }^{1}$ Centro de Astrobiología (CSIC-INTA), Ctra de Torrejón a Ajalvir, km 4, 28850 Torrejón de Ardoz, Madrid, Spain \\ e-mail: robertsj@inta.es \\ 2 Department of Physics \& Astronomy, University College London, Gower Street, London WC1E 6BT, UK \\ 3 School of Physics and Astronomy, University of Leeds, Leeds, LS2 9JT, UK \\ 4 Harvard-Smithsonian Astrophysical Observatory, 60 Garden Street, Cambridge, MA 02138, USA \\ 5 Escuela Universitaria de Óptica, Departamento de Matemática Aplicada (Biomatemática), Universidad Complutense de Madrid, \\ Avda. Arcos de Jalón s/n, 28037 Madrid, Spain \\ ${ }^{6}$ Laboratoire d'Astrophysique de Grenoble, UMR 5571-CNRS, Université Joseph Fourier, Grenoble, France
}

Received 7 August 2009 / Accepted 1 February 2010

ABSTRACT

\begin{abstract}
Context. Shock modelling predicts an electron density enhancement within the magnetic precursor of C-shocks. Previous observations of $\mathrm{SiO}, \mathrm{H}^{13} \mathrm{CO}^{+}, \mathrm{HN}^{13} \mathrm{C}$ and $\mathrm{H}^{13} \mathrm{CN}$ toward the young $\mathrm{L} 1448-\mathrm{mm}$ outflow showed an over-excitation of the ion fluid that was attributed to an electron density enhancement in the precursor.

Aims. We re-visit this interpretation and test if it still holds when we consider different source morphologies and kinetic temperatures for the observed molecules. To do this, we use updated collisional coefficients of $\mathrm{HN}^{13} \mathrm{C}$ and $\mathrm{SiO}$ with electrons in our excitation model. We also aim to give some insight on the spatial extent of the electron density enhancement around L1448-mm.

Methods. We estimate the opacities of $\mathrm{H}^{13} \mathrm{CO}^{+}$and $\mathrm{HN}^{13} \mathrm{C}$ by observing the $J=3 \rightarrow 2$ lines of rarer isotopologues. To model the excitation of the molecules, we use the large velocity gradient (LVG) approximation with updated collisional coefficients to i) re-analyse the observations toward the positions where the over-excitation of $\mathrm{H}^{13} \mathrm{CO}^{+}$has previously been observed [i.e. toward L1448-mm at offsets $(0,0)$ and $(0,-10)]$; and ii) to investigate if the electron density enhancement is still required for the cases of extended and compact emission, and for kinetic temperatures of up to $400 \mathrm{~K}$. We also report several lines of $\mathrm{SiO}, \mathrm{HN}^{13} \mathrm{C}$ and $\mathrm{H}^{13} \mathrm{CO}^{+}$ toward new positions around this outflow, to investigate the spatial extent of the over-excitation of the ions in L1448-mm.

Results. From the isotopologue observations, we find that the emission of $\mathrm{H}^{13} \mathrm{CO}^{+}$and $\mathrm{HN}^{13} \mathrm{C}$ from the precursor is optically thin if this emission is extended. Using the new collisional coefficients, an electron density enhancement is still needed to explain the excitation of $\mathrm{H}^{13} \mathrm{CO}^{+}$for extended emission and for gas temperatures of $\$ 400 \mathrm{~K}$ toward L1448-mm $(0,-10)$, and possibly also toward L1448-mm $(0,0)$. For compact emission the data cannot be fitted. We do not find any evidence for the over-excitation of the ion fluid toward the newly observed positions around L1448-mm.

Conclusions. The observed line emission of $\mathrm{SiO}, \mathrm{H}^{13} \mathrm{CO}^{+}$and $\mathrm{HN}^{13} \mathrm{C}$ toward $\mathrm{L} 1448-\mathrm{mm}(0,0)$ and $(0,-10)$ is consistent with an electron density enhancement in the precursor component, if this emission is spatially extended. This is also true for the case of high gas temperatures $(\$ 400 \mathrm{~K})$ toward the $(0,-10)$ offset. The electron density enhancement seems to be restricted to the southern, redshifted lobe of the L1448-mm outflow. Interferometric images of the line emission of these molecules are needed to confirm the spatial extent of the over-excitation of the ions and thus, of the electron density enhancement in the magnetic precursor of L1448-mm.
\end{abstract}

Key words. ISM: individual objects: L1448 - ISM: clouds - ISM: jets and outflows - ISM: molecules - shock waves

\section{Introduction}

C-shock waves associated with molecular outflows are belived to develop a thin region of enhanced fractional ionisation known as the "magnetic precursor" (Draine 1980; Flower et al. 1996; Flower \& Pineau des Forets 2003). Inside this region, the magnetic field is gradually compressed, forcing the ions to stream through the neutral gas, accelerating, compressing and heating this fluid before the neutral one. The subsequent ion-neutral velocity decoupling leads to the sputtering of dust grains, injecting large amounts of molecular material into the gas phase (e.g. Caselli et al. 1997; Schilke et al. 1997; Jiménez-Serra et al. 2008) The electron density is predicted to be enhanced within the magnetic precursor by a factor of $\sim 100$ due to the fluorescence UV photons generated after the collisional excitation of $\mathrm{H}_{2}$ molecules (Flower et al. 1996; Flower \& Pineau des Forets 2003).
It has been proposed that a narrow (line-width $\sim 0.5 \mathrm{~km} \mathrm{~s}^{-1}$ ) and very low velocity component of $\mathrm{SiO}$ detected towards the very young L1448-mm outflow, is the signature of the interaction of the magnetic precursor (Jiménez-Serra et al. 2004, hereafter JS04). The derived fractional abundance of $\mathrm{SiO}$ is of the order $10^{-11}$ in the velocity component of the precursor, an enhancement by a factor of 10 with respect to the upper limits measured in the quiescent gas of dark clouds ( $\$ 10^{-12}$ in L183 and L1448; Ziurys et al. 1989; Requena-Torres et al. 2007). Although the origin of this narrow $\mathrm{SiO}$ emission is still unclear, it has been suggested that the sputtering of dust grains at the precursor stage, is efficient enough to inject a considerable fraction of the grain mantles into the gas phase. As a consequence, bright and narrow $\mathrm{SiO}$ line emission is expected to arise from material whose central radial velocities are very similar to that of the ambient cloud (Jiménez-Serra et al. 2008, 2009). 
In correlation with the detection of narrow $\mathrm{SiO}$ emission, Jiménez-Serra et al. (2006, hereafter JS06) reported differences in the excitation of molecular ions, such as $\mathrm{H}^{13} \mathrm{CO}^{+}$, with respect to neutral molecules, such as $\mathrm{HN}^{13} \mathrm{C}$ and $\mathrm{H}^{13} \mathrm{CN}$, in the precursor component. In particular, the high- $J$ line emission of $\mathrm{H}^{13} \mathrm{CO}^{+}$is substantially brighter than that of $\mathrm{HN}^{13} \mathrm{C}$ toward those regions where the precursor has been detected. JS06 proposed that these differences, which cannot be accounted for by considering only molecular excitation by collisions with $\mathrm{H}_{2}$ and a single $\mathrm{H}_{2}$ density of few $\times 10^{5} \mathrm{~cm}^{-3}$ for the molecular gas, could be produced by the selective excitation of molecular ions by collisions with electrons within the precursor. This study established that the over-excitation of the ions is consistent with an electron density enhancement by a factor of $\sim 500$ in the precursor component toward L1448-mm $(0,0)$ and $(0,-10)$, which is similar to that predicted by $\mathrm{C}$-shock modelling at this dynamical time (Flower \& Pineau des Forets 2003).

However, the observed differences in excitation between the ion and the neutral molecules could be alternatively explained by opacity effects. Indeed, Frerking et al. (1979) and Cernicharo et al. (1984) showed anomalies in the large-scale line intensity emission of the hyperfine components of HNC and HCN toward the molecular dark clouds TMC-1 and L134N. The equal intensities measured for these components could be produced by the absorption of the emission arising from the dense cores by the less dense foreground material. This also applies to other high gas density tracers with high dipolar moment such as $\mathrm{HCO}^{+}$, since they also show similar behaviors for the line intensity ratios between its isotopologue species (Langer et al. 1978).

JS06 derived the electron density enhancement in the precursor component of L1448-mm by assuming that the $\mathrm{H}^{13} \mathrm{CO}^{+}$, the $\mathrm{HN}^{13} \mathrm{C}$ and the $\mathrm{H}^{13} \mathrm{CN}$ emission had thin to moderate optical depths. Unfortunately, the lack of even rarer isotopologue observations toward this outflow prevented, first, to determine the actual optical depths of this emission, and second, to clearly establish the origin of the over-excitation of the ions in L1448-mm.

In this paper we present new observations of $J=3 \rightarrow$ 2 lines of the rare isotopologues $\mathrm{HC}^{18} \mathrm{O}^{+}, \mathrm{H}^{13} \mathrm{C}^{18} \mathrm{O}^{+}, \mathrm{H}^{15} \mathrm{NC}$ and $\mathrm{H}^{15} \mathrm{~N}^{13} \mathrm{C}$, toward the regions where the over-excitation of the ions has been reported. In addition, we present new observations of the $J=3 \rightarrow 2$ lines of $\mathrm{HN}^{13} \mathrm{C}$ and $\mathrm{H}^{13} \mathrm{CO}^{+}$toward extra positions around the L1448-mm source, which we combine with previous observations toward these positions, observed by JS04, to derive the spatial extent of the over-excitation of $\mathrm{H}^{13} \mathrm{CO}^{+}$associated with the precursor. These observations will allow to prove that the anomalous excitation of the ions is not due to a large-scale scattering effect, but to a real enhancement of the local density of electrons at the early stages of the interaction of very young $\mathrm{C}$-shocks.

We also re-visit the analysis of JS06, using the same excitation model as JSO6 but including new collisional data of HNC and $\mathrm{SiO}$ with electrons (Faure et al. 2007; Varambhia et al. 2009, respectively), to test if the conclusions of JS06 still hold. The excitation model employed uses the large velocity gradient (LVG) approximation. We note that the L1448-mm outflow is likely the best object where the effects of collisional excitation by electrons on the molecular excitation can be directly tested. Furthermore, in our re-analysis we fully explore the effects of source morphology and kinetic gas temperature on our results.

In Sects. 2 and 3, we present the observations carried out with the JCMT telescope and describe the results. In Sect. 4, we estimate the opacities of $\mathrm{H}^{13} \mathrm{CO}^{+}$and $\mathrm{HN}^{13} \mathrm{C}$ toward the positions where the over-excitation of the ions has been reported, assuming both compact and extended source emission. In Sect. 5, we present the re-analysis of the LVG calculations of JS06 using the new collisional coefficients, considering different source morphologies and temperatures up to $400 \mathrm{~K}$. In this section we also analyse the data measured toward the new positions in L1448-mm to provide some insight into the spatial extent of the over-excitation of $\mathrm{H}^{13} \mathrm{CO}^{+}$. Finally, discussion and conclusions are presented in Sect. 6 .

\section{Observations}

We observed the $J=3 \rightarrow 2$ transition of the rare isotopologues $\mathrm{HC}^{18} \mathrm{O}^{+}, \mathrm{H}^{13} \mathrm{C}^{18} \mathrm{O}^{+}$and $\mathrm{H}^{15} \mathrm{~N}^{13} \mathrm{C}$ toward L1448-mm at offsets $(0,0)$ and $(0,-10)$, and $\mathrm{H}^{15} \mathrm{NC}$ toward L1448-mm $(0,0)$. The coordinates of the central position of the L1448-mm source are ( $\alpha=03^{\mathrm{h}} 25^{\mathrm{m}} 38.8, \delta=30^{\circ} 44^{\prime} 05^{\prime \prime} .4$ [J2000]). Finally, we measured the $J=3 \rightarrow 2$ lines of $\mathrm{H}^{13} \mathrm{CO}^{+}$and $\mathrm{HN}^{13} \mathrm{C}$ toward the offsets $(0,20),(20,0)$ and $(-20,0)$.

These lines, with frequencies ranging from 248 to $267 \mathrm{GHz}$, were observed with the James Clerk Maxwell Telescope (JCMT) at Mauna Kea (Hawaii), using the position switched observing mode and a reference (off) position of $\left(800^{\prime \prime}, 0\right)$ with respect to the central position. The ACSIS spectrometer provided a spectral resolution of $31 \mathrm{kHz}$, which corresponds to a velocity resolution of $\sim 0.04 \mathrm{~km} \mathrm{~s}^{-1}$ for the A3 receiver. The system temperatures ranged from $400 \mathrm{~K}$ to $700 \mathrm{~K}$.

The $J=3 \rightarrow 2$ lines of $\mathrm{H}^{13} \mathrm{CO}^{+}$and $\mathrm{HN}^{13} \mathrm{C}$ (at $\sim 260 \mathrm{GHz}$ ), observed with the IRAM $30 \mathrm{~m}$ telescope at Pico Veleta (Spain) toward L1448-mm $(0,0)$ and $(0,-10)$, were taken from JS06. The spectra of the $J=1 \rightarrow 0$ transitions of $\mathrm{H}^{13} \mathrm{CO}^{+}$and $\mathrm{HN}^{13} \mathrm{C}$, and of the $J=2 \rightarrow 1$ transition of $\mathrm{SiO}$ (at $\sim 87 \mathrm{GHz}$ ) toward L1448-mm $(0,20),(20,0)$ and $(-20,0)$, were also observed with this telescope, and have been previously published in JS04. See JS06 and JS04 for a full description of these observations.

Table 1 summarises the beam efficiencies and sizes, for all of the observations analysed in this paper. All the intensities were calibrated in units of antenna temperature $\left(T_{\mathrm{A}}^{*}\right)$.

\section{Results}

The line profiles of the $J=3 \rightarrow 2$ transitions of the rare isotopologues of $\mathrm{HCO}^{+}$and $\mathrm{HNC}$ observed toward L1448-mm $(0$, $0)$ and $(0,-10)$, are shown in Fig. 1 . The $J=3 \rightarrow 2$ lines of $\mathrm{H}^{13} \mathrm{CO}^{+}$and $\mathrm{HN}^{13} \mathrm{C}$ from JS06 are also plotted. The observed parameters of this emission are given in Table 2. Where detected, the line intensities are well above the $3 \sigma$ noise level. These data are used to calculate the upper limits on the opacity of the $\mathrm{H}^{13} \mathrm{CO}^{+}$and $\mathrm{HN}^{13} \mathrm{C}$ emission in Sect. 4 .

The $\mathrm{H}^{13} \mathrm{CO}^{+}$molecular line emission toward L1448-mm (0, 0 ) was fitted with a double Gaussian line profile, whose radial velocity components are centred at $V_{\mathrm{LSR}}=4.7 \mathrm{~km} \mathrm{~s}^{-1}$ (the ambient gas), and at $V_{\mathrm{LSR}}=5.2 \mathrm{~km} \mathrm{~s}^{-1}$ (the magnetic precursor component). These two velocity components were already reported by JS04 and JS06.

Toward L1448-mm $(0,-10)$, the $\mathrm{H}^{13} \mathrm{CO}^{+} J=3 \rightarrow 2$ emission shows a single peak centred at the precursor component with $V_{\mathrm{LSR}}=5.4 \mathrm{~km} \mathrm{~s}^{-1}$. The $\mathrm{HN}^{13} \mathrm{C} J=3 \rightarrow 2$ line, which is only detected toward L1448-mm $(0,0)$, has a single peak at the ambient gas velocity, $V_{\mathrm{LSR}}=4.7 \mathrm{~km} \mathrm{~s}^{-1}$. The $J=3 \rightarrow 2$ lines of the rare isotopologues $\mathrm{HC}^{18} \mathrm{O}^{+}, \mathrm{H}^{13} \mathrm{C}^{18} \mathrm{O}^{+}, \mathrm{H}^{15} \mathrm{NC}$ and $\mathrm{H}^{15} \mathrm{~N}^{13} \mathrm{C}$ were not detected above the $3 \sigma$ limit (see Table 2 ).

The profiles of the $\mathrm{SiO} J=2 \rightarrow 1$ lines, and of the $J=$ $3 \rightarrow 2$ and $1 \rightarrow 0$ transitions of $\mathrm{H}^{13} \mathrm{CO}^{+}$and $\mathrm{HN}^{13} \mathrm{C}$ toward the L1448-mm $(0,20),(20,0)$ and $(-20,0)$ offsets, are shown 
J. F. Roberts et al.: The magnetic precursor of L1448-mm

Table 1. Summary of beam efficiencies and FWHM beam sizes for all the observations analysed in this paper.

\begin{tabular}{|c|c|c|c|c|c|c|}
\hline Lines & Offset $\left({ }^{\prime \prime}\right)$ & Frequency $(\mathrm{GHz})$ & Telescope & $\eta$ & beam size $\left({ }^{\prime \prime}\right)$ & Reference \\
\hline $\begin{array}{l}\mathrm{HC}^{18} \mathrm{O}^{+}(3-2), \mathrm{H}^{13} \mathrm{C}^{18} \mathrm{O}^{+}(3-2), \\
\mathrm{H}^{15} \mathrm{~N}^{13} \mathrm{C}(3-2), \mathrm{H}^{15} \mathrm{NC}(3-2)\end{array}$ & $(0,0),(0,-10)$ & $248-267$ & JCMT & 0.69 & 21 & This work \\
\hline $\mathrm{H}^{13} \mathrm{CO}^{+}(3-2), \mathrm{HN}^{13} \mathrm{C}(3-2)$ & $(0,20),(20,0),(-20,0)$ & $\sim 260$ & JCMT & 0.69 & 21 & This work \\
\hline $\begin{array}{l}\mathrm{SiO}(2-1), \mathrm{HN}^{13} \mathrm{C}(1-0), \\
\mathrm{H}^{13} \mathrm{CO}^{+}(1-0)\end{array}$ & $(0,0),(0,-10)$ & $\sim 90$ & IRAM & 0.82 & 28 & JS06 \\
\hline $\mathrm{SiO}(3-2)$ & $(0,0),(0,-10)$ & $\sim 130$ & IRAM & 0.74 & 19 & JS06 \\
\hline $\begin{array}{l}\mathrm{SiO}(5-4), \mathrm{HN}^{13} \mathrm{C}(3-2), \\
\mathrm{H}^{13} \mathrm{CO}^{+}(3-2)\end{array}$ & $(0,0),(0,-10)$ & $217-260$ & IRAM & 0.52 & 11 & JS06 \\
\hline $\mathrm{HN}^{13} \mathrm{C}(4-3), \mathrm{H}^{13} \mathrm{CO}^{+}(4-3)$ & $(0,0),(0,-10)$ & $\sim 350$ & JCMT & 0.63 & 14 & JS06 \\
\hline $\mathrm{H}^{13} \mathrm{CO}^{+}(1-0), \mathrm{HN}^{13} \mathrm{C}(1-0)$ & $(0,20),(20,0),(-20,0)$ & $\sim 87$ & IRAM & 0.82 & 28 & JS04 \\
\hline
\end{tabular}

Table 2. Observed parameters of the $J=3 \rightarrow 2$ lines of $\mathrm{H}^{13} \mathrm{CO}^{+}, \mathrm{HC}^{18} \mathrm{O}^{+}, \mathrm{H}^{13} \mathrm{C}^{18} \mathrm{O}^{+}, \mathrm{HN}^{13} \mathrm{C}, \mathrm{H}^{15} \mathrm{NC}$ and $\mathrm{H}^{15} \mathrm{~N}^{13} \mathrm{C}$ toward $\mathrm{L} 1448$-mm $(0,0)$ and $(0,-10)$.

\begin{tabular}{|c|c|c|c|c|c|c|}
\hline \multirow[b]{2}{*}{ Line } & \multicolumn{3}{|c|}{$(0,0)$} & \multicolumn{3}{|c|}{$(0,-10)$} \\
\hline & $\begin{array}{c}V_{\mathrm{LSR}} \\
\left(\mathrm{km} \mathrm{s}^{-1}\right)\end{array}$ & $\begin{array}{c}\Delta v \\
\left(\mathrm{~km} \mathrm{~s}^{-1}\right)\end{array}$ & $\begin{array}{l}T_{\mathrm{A}}^{*} \\
(\mathrm{~K})\end{array}$ & $\begin{array}{c}V_{\mathrm{LSR}} \\
\left(\mathrm{km} \mathrm{s}^{-1}\right)\end{array}$ & $\begin{array}{c}\Delta v \\
\left(\mathrm{~km} \mathrm{~s}^{-1}\right)\end{array}$ & $\begin{array}{l}T_{\mathrm{A}}^{*} \\
(\mathrm{~K})\end{array}$ \\
\hline \multirow[t]{2}{*}{$\mathrm{H}^{13} \mathrm{CO}^{+a}$} & $4.5(2)$ & $0.9(3)$ & $0.23(9)$ & $\sim 4.7$ & & $\$ 0.168$ \\
\hline & $5.35(1)$ & $0.71(3)$ & 2.21(9) & $5.52(4)$ & $0.90(8)$ & $0.65(6)$ \\
\hline \multirow[t]{2}{*}{$\mathrm{HC}^{18} \mathrm{O}^{+}$} & $\sim 4.7$ & ... & $\lesssim 0.195^{b}$ & $\sim 4.7$ & ... & $\lesssim 0.195$ \\
\hline & $\sim 5.2$ & $\ldots$ & $\lessgtr 0.195$ & $\sim 5.2$ & $\ldots$ & $\lesssim 0.195$ \\
\hline \multirow[t]{2}{*}{$\mathrm{H}^{13} \mathrm{C}^{18} \mathrm{O}^{+}$} & $\sim 4.7$ & $\ldots$ & $\lesssim 0.090$ & $\sim 4.7$ & $\ldots$ & $\lesssim 0.087$ \\
\hline & $\sim 5.2$ & $\ldots$ & $\lesssim 0.090$ & $\sim 5.2$ & $\ldots$ & $\lesssim 0.087$ \\
\hline \multirow[t]{2}{*}{$\mathrm{HN}^{13} \mathrm{C}^{a}$} & $4.75(5)$ & $0.6(1)$ & $0.35(9)$ & $\sim 4.7$ & $\ldots$ & $\lessgtr 0.261$ \\
\hline & $\sim 5.2$ & $\ldots$ & $\lessgtr 0.237$ & $\sim 5.2$ & $\ldots$ & $\lesssim 0.261$ \\
\hline \multirow[t]{2}{*}{$\mathrm{H}^{15} \mathrm{NC}$} & $\sim 4.7$ & $\ldots$ & $\lessgtr 0.093$ & $\ldots$ & $\ldots$ & $\ldots$ \\
\hline & $\sim 5.2$ & $\ldots$ & $\lessgtr 0.093$ & $\ldots$ & $\ldots$ & $\ldots$ \\
\hline \multirow[t]{2}{*}{$\mathrm{H}^{15} \mathrm{~N}^{13} \mathrm{C}$} & $\sim 4.7$ & $\ldots$ & $\$ 0.198$ & $\sim 4.7$ & $\ldots$ & $\lessgtr 0.207$ \\
\hline & $\sim 5.2$ & $\ldots$ & $\lessgtr 0.198$ & $\sim 5.2$ & $\ldots$ & $\lesssim 0.207$ \\
\hline
\end{tabular}

Notes. ${ }^{(a)}$ These molecular line transitions were taken from JS06. ${ }^{(b)}$ The upper limits of the line intensities correspond to the $3 \sigma$ noise level.

Table 3. Observed parameters of the $J=2 \rightarrow 1$ line of $\mathrm{SiO}$, and of the $J=3 \rightarrow 2$ and $J=1 \rightarrow 0$ transitions of $\mathrm{H}^{13} \mathrm{CO}^{+}$and $\mathrm{HN}^{13} \mathrm{C}$ toward $\operatorname{L} 1448-\mathrm{mm}(0,20),(20,0)$ and $(-20,0)$.

\begin{tabular}{|c|c|c|c|c|c|c|c|c|c|}
\hline \multirow[b]{2}{*}{ Line } & \multicolumn{3}{|c|}{$(0,20)$} & \multicolumn{3}{|c|}{$(20,0)$} & \multicolumn{3}{|c|}{$(-20,0)$} \\
\hline & $\begin{array}{c}V_{\mathrm{LSR}} \\
\left(\mathrm{km} \mathrm{s}^{-1}\right)\end{array}$ & $\begin{array}{c}\Delta v \\
\left(\mathrm{~km} \mathrm{~s}^{-1}\right)\end{array}$ & $\begin{array}{l}T_{\mathrm{A}}^{*} \\
(\mathrm{~K})\end{array}$ & $\begin{array}{c}V_{\mathrm{LSR}} \\
\left(\mathrm{km} \mathrm{s}^{-1}\right)\end{array}$ & $\begin{array}{c}\Delta v \\
\left(\mathrm{~km} \mathrm{~s}^{-1}\right)\end{array}$ & $\begin{array}{l}T_{\mathrm{A}}^{*} \\
(\mathrm{~K})\end{array}$ & $\begin{array}{c}V_{\mathrm{LSR}} \\
\left(\mathrm{km} \mathrm{s}^{-1}\right)\end{array}$ & $\begin{array}{c}\Delta v \\
\left(\mathrm{~km} \mathrm{~s}^{-1}\right)\end{array}$ & $\begin{array}{l}T_{\mathrm{A}}^{*} \\
(\mathrm{~K})\end{array}$ \\
\hline \multirow[t]{2}{*}{$\mathrm{SiO}(2 \rightarrow 1)^{a}$} & $\sim 4.7$ & 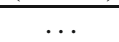 & $\$ 0.027^{b}$ & $\sim 4.7$ & & $\$ 0.030$ & $\sim 4.7$ & & $\lesssim 0.036$ \\
\hline & $\sim 5.2$ & . & $\lesssim 0.027$ & $5.07(6)$ & $0.5(1)$ & $0.04(1)$ & $5.29(4)$ & $0.51(7)$ & $0.06(1)$ \\
\hline \multirow[t]{2}{*}{$\mathrm{H}^{13} \mathrm{CO}^{+}(1 \rightarrow 0)^{a}$} & $4.597(3)$ & $0.625(7)$ & $0.75(2)$ & $4.645(4)$ & $0.590(9)$ & $0.74(2)$ & $4.606(3)$ & $0.87(2)$ & $0.49(3)$ \\
\hline & $5.126(0)$ & $0.82(1)$ & $0.57(2)$ & $5.254(0)$ & $0.89(1)$ & $0.68(2)$ & $5.318(1)$ & $0.72(1)$ & $0.85(3)$ \\
\hline \multirow[t]{2}{*}{$\mathrm{H}^{13} \mathrm{CO}^{+}(3 \rightarrow 2)$} & $4.5(1)$ & $0.5(3)$ & $0.20(6)$ & $\sim 4.7$ & & $\lesssim 0.14$ & $\sim 4.7$ & & $\lesssim 0.09$ \\
\hline & $5.33(7)$ & $0.7(2)$ & $0.36(5)$ & $5.2(1)$ & $1.0(3)$ & $0.22(5)$ & $5.3(2)$ & $0.4(2)$ & $0.14(3)$ \\
\hline \multirow[t]{2}{*}{$\mathrm{HN}^{13} \mathrm{C}(1 \rightarrow 0)^{a}$} & $4.625(6)$ & $0.80(1)$ & $0.52(1)$ & $4.6(1)$ & $0.8(1)$ & $0.57(1)$ & $4.89(1)$ & $0.86(2)$ & $0.84(1)$ \\
\hline & $5.224(2)$ & $0.80(5)$ & $0.15(1)$ & $5.2(1)$ & $0.9(1)$ & $0.40(1)$ & $5.58(4)$ & $0.63(6)$ & $0.21(1)$ \\
\hline \multirow[t]{2}{*}{$\mathrm{HN}^{13} \mathrm{C}(3 \rightarrow 2)$} & $\sim 4.7$ & .. & $\lessgtr 0.07$ & $\sim 4.7$ & 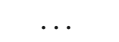 & $\lessgtr 0.075$ & $4.5(2)$ & $0.7(2)$ & $0.09(2)$ \\
\hline & $\sim 5.2$ & $\ldots$ & $\lesssim 0.07$ & $\sim 5.2$ & $\ldots$ & $\lessgtr 0.075$ & $\sim 5.2$ & $\ldots$ & $\lesssim 0.07$ \\
\hline
\end{tabular}

Notes. ${ }^{(a)}$ These molecular line transitions were taken from JS04. ${ }^{(b)}$ The upper limits of the line intensities correspond to the $3 \sigma$ noise level.

in Fig. 2. The observed parameters are given in Table 3, with all detections above the $3 \sigma$ noise level.

Narrow, single-peaked $\mathrm{SiO} J=2 \rightarrow 1$ emission is detected toward L1448-mm (-20, 0), and very faintly toward L1448$\mathrm{mm}(20,0)$. These lines are centred at the velocity of the precursor component $\left(\sim 5.2 \mathrm{~km} \mathrm{~s}^{-1}\right)$, and show line-widths of $\sim$ $0.5 \mathrm{~km} \mathrm{~s}^{-1}$ (Table 3). The line profiles of $\mathrm{H}^{13} \mathrm{CO}^{+} J=1 \rightarrow 0$ and $\mathrm{HN}^{13} \mathrm{C} J=1 \rightarrow 0$ are fitted by double Gaussian profiles, with line widths of $0.6-0.8 \mathrm{~km} \mathrm{~s}^{-1}$ for each component. Toward L 1448-mm $(0,20)$ and $(20,0)$, the $\mathrm{H}^{13} \mathrm{CO}^{+} J=1 \rightarrow 0$ lines show their maximum emission at the ambient velocity component with $V_{\text {LSR }}=4.7 \mathrm{~km} \mathrm{~s}^{-1}$. However, toward L1448 $(-20,0)$ where the detection of narrow $\mathrm{SiO}$ is more evident, $\mathrm{H}^{13} \mathrm{CO}^{+} J=$ $1 \rightarrow 0$ peaks at the precursor component with $V_{\mathrm{LSR}}=5.2 \mathrm{~km} \mathrm{~s}^{-1}$. The peak emission of the $\mathrm{HN}^{13} \mathrm{C} J=1 \rightarrow 0$ lines are all centred at the velocity of the ambient gas $\left(V_{\mathrm{LSR}} \sim 4.7 \mathrm{~km} \mathrm{~s}^{-1}\right)$, except toward L1448-mm $(-20,0)$ where this emission is red-shifted to $\sim 4.9 \mathrm{~km} \mathrm{~s}^{-1}$ (Table 3).

The $J=3 \rightarrow 2$ lines of $\mathrm{H}^{13} \mathrm{CO}^{+}$show single peaked line profiles centred at $\sim 5.2 \mathrm{~km} \mathrm{~s}^{-1}$ toward L1448-mm $(20,0)$ and 


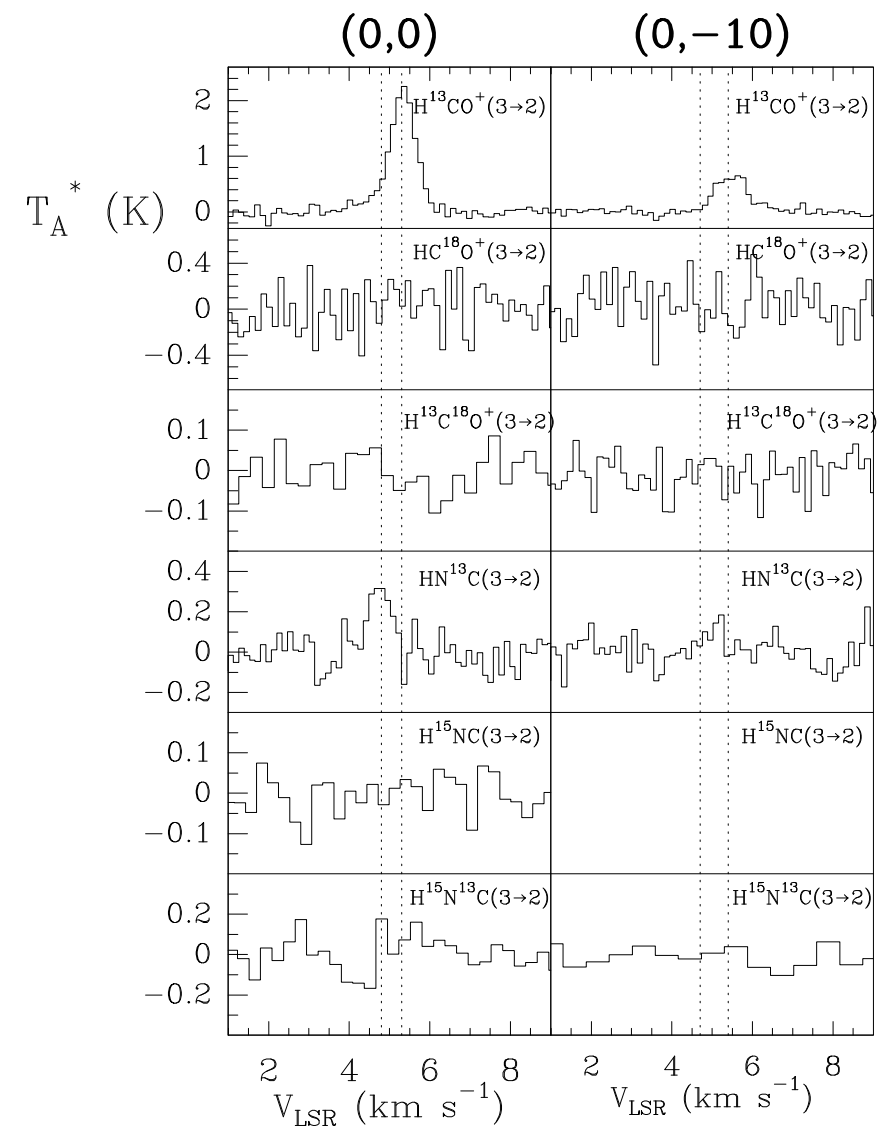

Fig. 1. Observations of the $J=3 \rightarrow 2$ line of several isotopologue species of $\mathrm{HCO}^{+}$and $\mathrm{HNC}$ toward the L1448-mm $(0,0)$ and $(0,-10)$ positions. Offsets, in arcseconds, are shown in the upper part of the columns. The vertical dotted lines show the ambient gas centred at $4.7 \mathrm{~km} \mathrm{~s}^{-1}$, and the precursor component at 5.2 and $5.4 \mathrm{~km} \mathrm{~s}^{-1}$ toward L1448-mm $(0,0)$ and $(0,-10)$, respectively (see JS06).

$(-20,0)$, while toward L1448-mm $(0,20)$, a double Gaussian line profile can roughly be fitted. This behaviour was also reported by JS06 toward L1448-mm $(0,0)$, where the $J=3 \rightarrow 2$ and $J=4 \rightarrow 3$ lines of $\mathrm{H}^{13} \mathrm{CO}^{+}$also show faint emission arising from the ambient gas. As shown by JS06, this emission is consistent with the excitation of $\mathrm{H}^{13} \mathrm{CO}^{+}$by collisions with only $\mathrm{H}_{2}$, assuming an $\mathrm{H}_{2}$ density of $\sim 10^{5} \mathrm{~cm}^{-3}$.

Toward $(20,0)$ and $(-20,0)$, the detection of $\mathrm{H}^{13} \mathrm{CO}^{+}$emission only in the precursor component suggests that the ions could have already been accelerated within the shock to larger redshifted velocities. This is more apparent toward $(-20,0)$ where all molecular lines have their peak emission slightly red-shifted to larger velocities compared to other positions. As proposed by JS06, this velocity shift could be understood as an evolutionary effect in which the molecular material toward L1448-mm $(-20$, 0 ) is at a later dynamical time within the C-shock. This is also supported by the clear detection of narrow $\mathrm{SiO}$ toward this position. We finally note that no signal above the $3 \sigma$ noise level was detected for the $\mathrm{HN}^{13} \mathrm{C} J=3 \rightarrow 2$ lines at any position in L1448-mm for the precursor component. In Sect. 5.2, all these data will be analysed to establish the spatial extent of the overexcitation of $\mathrm{H}^{13} \mathrm{CO}^{+}$in L1448-mm, and its implications for the magnetic precursor scenario.

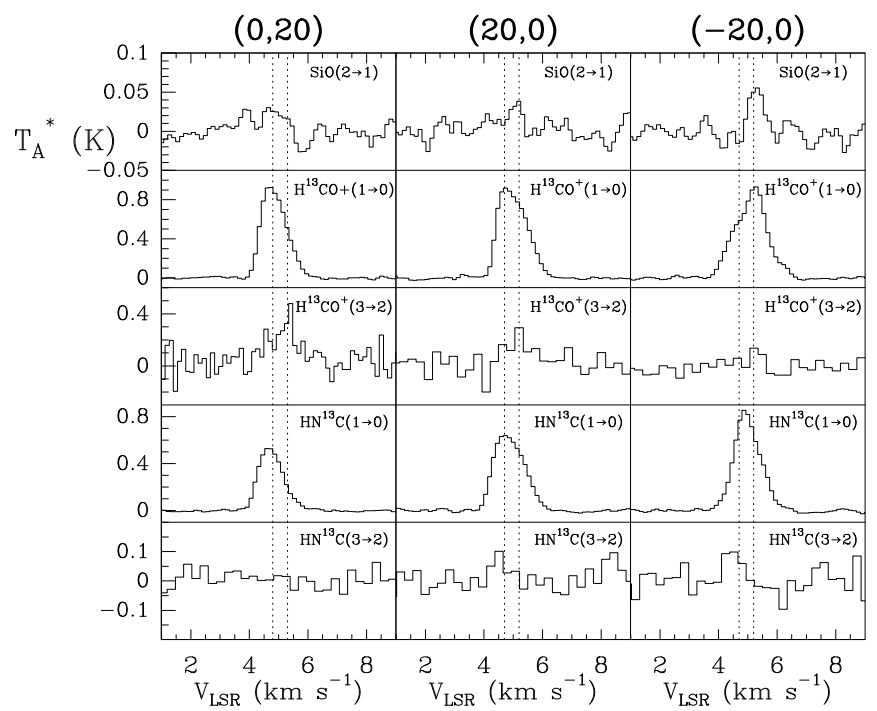

Fig. 2. Observations of the $\mathrm{SiO} J=2 \rightarrow 1$ line emission and of the $J=3 \rightarrow 2$ and $1 \rightarrow 0$ transitions of $\mathrm{H}^{13} \mathrm{CO}^{+}$and $\mathrm{HN}^{13} \mathrm{C}$ toward the L1448-mm $(0,20),(20,0)$ and $(-20,0)$ positions. Offsets, in arcseconds, are shown in the upper part of the columns. The vertical dotted lines show the ambient gas at $4.7 \mathrm{~km} \mathrm{~s}^{-1}$ and the precursor component at $5.2 \mathrm{~km} \mathrm{~s}^{-1}$.

Table 4. Isotopic abundance ratios for the local ISM taken from Wilson (1999).

\begin{tabular}{cc}
\hline \hline Species & Ratio $(R)$ \\
\hline$\frac{\mathrm{HC}^{18} \mathrm{O}^{+}}{\mathrm{H}^{13} \mathrm{CO}^{+}}=\frac{\left[{ }^{12} \mathrm{C}\right]}{\left[{ }^{13} \mathrm{C}\right]} \times \frac{\left[{ }^{18} \mathrm{O}\right]}{\left[{ }^{16} \mathrm{O}\right]}$ & $\frac{69}{577}$ \\
$\frac{\mathrm{H}^{13} \mathrm{C}^{18} \mathrm{O}^{+}}{\mathrm{H}^{13} \mathrm{CO}^{+}}=\frac{\left[{ }^{18} \mathrm{O}\right]}{\left[{ }^{16} \mathrm{O}\right]}$ & $\frac{1}{577}$ \\
$\frac{\mathrm{H}^{15} \mathrm{NC}}{\mathrm{HN}^{13} \mathrm{C}}=\frac{\left[{ }^{15} \mathrm{~N}\right]}{\left[{ }^{14} \mathrm{~N}\right]} \times \frac{\left[{ }^{12} \mathrm{C}\right]}{\left[{ }^{13} \mathrm{C}\right]}$ & $\frac{69}{388}$ \\
$\frac{\mathrm{H}^{15} \mathrm{~N}^{13} \mathrm{C}}{\mathrm{HN}^{13} \mathrm{C}}=\frac{\left[{ }^{15} \mathrm{~N}\right]}{\left[{ }^{14} \mathrm{~N}\right]}$ & $\frac{1}{388}$ \\
\hline
\end{tabular}

\section{Calculation of opacities}

In order to rule out the possibility that the over-excitation of $\mathrm{H}^{13} \mathrm{CO}^{+}$is due to large opacity effects, we need to prove that the emission lines of $\mathrm{H}^{13} \mathrm{CO}^{+}$and $\mathrm{HN}^{13} \mathrm{C}$ reported by JS06 are optically thin. In addition, low optical depths associated with the $\mathrm{H}^{13} \mathrm{CO}^{+}$and $\mathrm{HN}^{13} \mathrm{C}$ emission toward L1448-mm will fully justify the use of the LVG approximation as the molecular excitation model to derive the electron density enhancement predicted at the precursor stage.

We can estimate the optical depths of the $\mathrm{H}^{13} \mathrm{CO}^{+}$and $\mathrm{HN}^{13} \mathrm{C} J=3 \rightarrow 2$ lines, $\tau_{32}$, toward L1448-mm $(0,0)$ and $(0$, $-10)$ using the observed isotopic line intensity ratios (Choi et al. 1993):

$\frac{T_{\mathrm{B}, \mathrm{rare}}}{T_{\mathrm{B}, 32}}=\frac{1-\mathrm{e}^{-R \tau_{32}}}{1-\mathrm{e}^{-\tau_{32}}}$.

This equation assumes equal excitation temperature for both isotopologues. Here, $T_{\mathrm{B}, 32}$ is the brightness temperature of the $\mathrm{H}^{13} \mathrm{CO}^{+} J=3 \rightarrow 2$ or $\mathrm{HN}^{13} \mathrm{C} J=3 \rightarrow 2$ line, and $T_{\mathrm{B} \text {,rare }}$ is the brightness temperature of the $J=3 \rightarrow 2$ line of the corresponding rarer isotopologue (either $\mathrm{HC}^{18} \mathrm{O}^{+}$or $\mathrm{H}^{13} \mathrm{C}^{18} \mathrm{O}^{+}$for $\mathrm{H}^{13} \mathrm{CO}^{+}$, or $\mathrm{H}^{15} \mathrm{NC}$ or $\mathrm{H}^{15} \mathrm{~N}^{13} \mathrm{C}$ for $\mathrm{HN}^{13} \mathrm{C}$ ). $R$ is the typical isotopic abundance ratio observed in the local interstellar medium, 
Table 5. Optical depths of the $\mathrm{H}^{13} \mathrm{CO}^{+} J=3 \rightarrow 2$ lines toward L1448-mm $(0,0)$ and $(0,-10)$ derived from different isotopologue ratios for the ambient (amb.) and the precursor (prec.) components, and for different source morphologies.

\begin{tabular}{|c|c|c|c|c|c|}
\hline \multicolumn{6}{|c|}{$\overline{(0,0)}$} \\
\hline Ratio used & Component & $T_{\mathrm{B}, \mathrm{rare}} / T_{\mathrm{B}, 32}$ & $\tau_{32}\left(\mathrm{H}^{13} \mathrm{CO}^{+}\right)$ & $T_{\mathrm{B}, \mathrm{rare}} / T_{\mathrm{B}, 32}$ & $\tau_{32}\left(\mathrm{H}^{13} \mathrm{CO}^{+}\right)$ \\
\hline \multirow{3}{*}{$\mathrm{HC}^{18} \mathrm{O}^{+} / \mathrm{H}^{13} \mathrm{CO}^{+}$} & \multirow{3}{*}{$\begin{array}{l}\text { Amb. } \\
\text { Prec. }\end{array}$} & \multicolumn{2}{|c|}{ Source size $5^{\prime \prime}$} & \multicolumn{2}{|c|}{ Extended source } \\
\hline & & $\$ 2.4$ & $\ldots$ & $\$ 0.9$ & $\lesssim 16$ \\
\hline & & $\lesssim 0.25$ & $\lesssim 2.1$ & $\lesssim 0.09$ & $\lesssim 0.59^{a}$ \\
\hline \multirow[t]{2}{*}{$\mathrm{H}^{13} \mathrm{C}^{18} \mathrm{O}^{+} / \mathrm{H}^{13} \mathrm{CO}^{+}$} & Amb. & $\lesssim 1.1$ & $\ldots$ & $\lesssim 0.4$ & $\lesssim 290$ \\
\hline & Prec. & $\lesssim 0.11$ & $\$ 67$ & $\lesssim 0.04$ & $\lesssim 24$ \\
\hline \multicolumn{6}{|c|}{$\overline{(0,-10)}$} \\
\hline \multirow[t]{2}{*}{ Ratio used } & Component & $T_{\mathrm{B}, \text { rare }} / T_{\mathrm{B}, 32}$ & $\tau_{32}\left(\mathrm{H}^{13} \mathrm{CO}^{+}\right)$ & $T_{\mathrm{B}, \mathrm{rare}} / T_{\mathrm{B}, 32}$ & $\tau_{32}\left(\mathrm{H}^{13} \mathrm{CO}^{+}\right)$ \\
\hline & & \multicolumn{2}{|c|}{ Source size $5^{\prime \prime}$} & \multicolumn{2}{|c|}{ Extended source } \\
\hline \multirow[t]{2}{*}{$\mathrm{HC}^{18} \mathrm{O}^{+} / \mathrm{H}^{13} \mathrm{CO}^{+}$} & Amb. & $\$ 3.1$ & $\ldots$ & $\lesssim 1.1$ & $\ldots$ \\
\hline & Prec. & $\lesssim 0.85$ & $\lesssim 16$ & $\lesssim 0.3$ & $\$ 2.8$ \\
\hline \multirow[t]{2}{*}{$\mathrm{H}^{13} \mathrm{C}^{18} \mathrm{O}^{+} / \mathrm{H}^{13} \mathrm{CO}^{+}$} & Amb. & $\lessgtr 1.4$ & ... & $\lessgtr 0.5$ & $\$ 390$ \\
\hline & Prec. & $\lessgtr 0.38$ & $\$ 280$ & $\lessgtr 0.1$ & $\lesssim 83$ \\
\hline
\end{tabular}

Notes. ${ }^{(a)}$ This value of $\tau\left(\mathrm{H}^{13} \mathrm{CO}^{+}\right)$was estimated assuming $R=\frac{40}{577}$, rather than $R=\frac{69}{577}$ (see text for explanation).

values for which are given in Table 4 (Wilson 1999). These values are averages for the local ISM.

To estimate the brightness temperature ratios from the values of $T_{\mathrm{A}}^{*}$ given in Table 2, we need to know the source morphology. Since the morphology of the precursor component of the $\mathrm{H}^{13} \mathrm{CO}^{+}$and $\mathrm{HN}^{13} \mathrm{C}$ emission is unknown, we assume two possible extreme cases: compact emission with a source size of $5^{\prime \prime}$, and extended emission.

For the source size of $5^{\prime \prime}$, the line intensities in units of $T_{\mathrm{A}}^{*}$ are converted to brightness temperatures by the formula $T_{\mathrm{B}}=\frac{T_{\mathrm{A}}^{*}}{\eta}\left(\theta_{\text {source }}^{2}+\theta_{\text {beam }}^{2}\right) / \theta_{\text {source }}^{2}$ where $\theta_{\text {source }}$ is the source size, $\theta_{\text {beam }}$ is the beam size and $\eta$ is the beam efficiency. Values for $\theta_{\text {beam }}$ and $\eta$ are given in Table 1 . For the extended source case, the brightness temperatures are approximated by $T_{\mathrm{A}}^{*}$.

The estimated line intensity ratios and optical depths for the $\mathrm{H}^{13} \mathrm{CO}^{+}$and $\mathrm{HN}^{13} \mathrm{C} J=3 \rightarrow 2$ emission are given in Tables 5 and 6 , respectively, for the different source morphologies and isotopologue ratios considered.

An inspection of Tables 5 and 6 clearly shows that, many line ratios are larger than unity due to the lack of sensitivity of our observations for the rarer isotopologues. Under these conditions Eq. (1) cannot be used to estimate the opacities.

From Tables 5 and 6 we draw the following conclusions:

- Ambient components: for the ambient components of the $\mathrm{H}^{13} \mathrm{CO}^{+} J=3 \rightarrow 2$ emission, the data is not sensitive enough to provide useful constraints. For $\mathrm{HN}^{13} \mathrm{C} J=3 \rightarrow 2$ toward $(0,0)$, assuming an extended source, the ambient component is relatively optically thin $\left(\tau_{32} \lesssim 1\right.$. 1 , derived from the $\mathrm{H}^{15} \mathrm{NC} / \mathrm{HN}^{13} \mathrm{C}$ ratio). Since the line emission from the ambient component has indeed been observed to be extended (Bachiller et al. 1990; Curiel et al. 1999), from our data on $\mathrm{HN}^{13} \mathrm{C} J=3 \rightarrow 2$ we conclude that the emission from this component is optically thin, as assumed by JS06. The ambient component will not be further considered in this paper.

- $\mathbf{H}^{13} \mathbf{C O}^{+} J=3 \rightarrow 2$ precursor component toward $(\mathbf{0 , 0})$ : if the source size is $\geq 5^{\prime \prime}$, this line is relatively optically thin $\left(\tau_{32} \lesssim 2.1\right.$, using the estimate derived from the $\mathrm{HC}^{18} \mathrm{O}^{+} / \mathrm{H}^{13} \mathrm{CO}^{+}$ratio). For the extended source case, the $\mathrm{HC}^{18} \mathrm{O}^{+} / \mathrm{H}^{13} \mathrm{CO}^{+}$ratio has an upper limit of 0.09 , which results in a negative solution for the optical depth for the assumed value of $R$. This is because Eq. (1) only has a positive solution for $\tau_{32}$ for $R \lesssim T_{\mathrm{B}, \text { rare }} / T_{\mathrm{B}, 32} \lesssim 1$. Even if we consider the lower limit of the $\mathrm{H}^{13} \mathrm{CO}^{+} J=3 \rightarrow 2$ line intensity, corresponding to the $1 \sigma$ noise level, the upper limit of $T_{\mathrm{B}, \text { rare }} / T_{\mathrm{B}, 32}$ remains less than $R$. This suggests that the actual isotopic ratios in L1448 deviate from the averaged values of $R=69 / 577$ in Table 4. Significant variations in the isotopic ratios in nearby dark clouds have been recently found. Indeed, the ${ }^{12} \mathrm{C} /{ }^{13} \mathrm{C}$ ratio has been estimated to vary from $\sim 40$ to $\sim 90$ within the local ISM (Casassus et al. 2005). If we assume a extreme value of ${ }^{12} \mathrm{C} /{ }^{13} \mathrm{C}=40, R$ decreases to $\sim 0.07$ providing an optical depth of $\tau_{32} \lesssim 0.6$. Therefore, our data suggest a rather low ${ }^{12} \mathrm{C} /{ }^{13} \mathrm{C}$ ratio and/or a high ${ }^{16} \mathrm{O} /{ }^{18} \mathrm{O}$ ratio in the L1448 molecular cloud if the emission is extended. In summary, the emission of $\mathrm{H}^{13} \mathrm{CO}^{+} J=3 \rightarrow 2$ for the precursor toward $(0,0)$ is optically thin even if it arises from a small source.

- $\mathbf{H}^{13} \mathbf{C O}^{+} J=3 \rightarrow 2$ precursor component toward $(\mathbf{0},-\mathbf{1 0})$ : if the source is extended, this line is also relatively optically thin $\left(\tau_{32} \lesssim 2.8\right.$, derived from the $\mathrm{HC}^{18} \mathrm{O}^{+} / \mathrm{H}^{13} \mathrm{CO}^{+}$ratio). For a source size of $5^{\prime \prime}$, we can only constrain $\tau_{32}$ to $\lesssim 16$.

$-\mathbf{H N}^{13} \mathbf{C} J=3 \rightarrow 2$ precursor component toward $(0,0)$ and $(\mathbf{0}, \mathbf{- 1 0})$ : for both geometries we cannot constrain the optical depths due to the lack of sensitivity of our observations. However, for a source of size 5", we find in Sect. 5.1.3 that our LVG model cannot reproduce the observed intensities of $\mathrm{HN}^{13} \mathrm{C}$, implying that the emission should be extended.

\section{Excitation differences between ion and neutral molecular fluids}

\subsection{Revisiting the ion and neutral molecular excitation analysis toward L1448-mm: new collisional data and the effect of temperature and source size}

JS06 proposed that the over-excitation of the $\mathrm{H}^{13} \mathrm{CO}^{+}$molecular ions observed in the precursor component toward L1448-mm $(0$, $0)$ and $(0,-10)$ could be explained by an enhanced fractional abundance of electrons of $\sim 5 \times 10^{-5}$. We need to check if this conclusion still holds when using the newly released collisional coefficients of $\mathrm{SiO}$ and $\mathrm{HNC}$ with electrons (Varambhia et al. 2009; Faure et al. 2007).

In addition, JS06 assumed that the kinetic temperature of the gas was low $\left(21 \mathrm{~K}\right.$, estimated from $\mathrm{NH}_{3}$ observations; Curiel 
Table 6. Optical depths of the $\mathrm{HN}^{13} \mathrm{C} J=3 \rightarrow 2$ line toward L1448-mm $(0,0)$ and $(0,-10)$ derived from different isotopologue ratios for the ambient (amb.) and the precursor (prec.) components, and for different source morphologies.

\begin{tabular}{|c|c|c|c|c|c|}
\hline \multicolumn{6}{|c|}{$\overline{(0,0)}$} \\
\hline Ratio used & Component & $T_{\mathrm{B}, \text { rare }} / T_{\mathrm{B}, 32}$ & $\tau_{32}\left(\mathrm{HN}^{13} \mathrm{C}\right)$ & $T_{\mathrm{B}, \text { rare }} / T_{\mathrm{B}, 32}$ & $\tau_{32}\left(\mathrm{HN}^{13} \mathrm{C}\right)$ \\
\hline \multirow{3}{*}{$\mathrm{H}^{15} \mathrm{NC} / \mathrm{HN}^{13} \mathrm{C}$} & & \multicolumn{2}{|c|}{ Source size $5^{\prime \prime}$} & \multicolumn{2}{|c|}{ Extended source } \\
\hline & \multirow{2}{*}{$\begin{array}{l}\text { Amb. } \\
\text { Prec. }\end{array}$} & $\$ 0.74$ & $\$ 7.6$ & $\$ 0.3$ & $\lesssim 1.1$ \\
\hline & & $\sim 1.1$ & $\ldots$ & $\sim 0.4$ & $\sim 2.5$ \\
\hline \multirow[t]{2}{*}{$\mathrm{H}^{15} \mathrm{~N}^{13} \mathrm{C} / \mathrm{HN}^{13} \mathrm{C}$} & Amb. & $\lesssim 1.6$ & $\ldots$ & $\lesssim 0.6$ & $\$ 320$ \\
\hline & Prec. & $\sim 2.3$ & $\ldots$ & $\sim 0.8$ & $\sim 700$ \\
\hline \multicolumn{6}{|c|}{$\overline{(0,-10)}$} \\
\hline Ratio used & Component & $T_{\mathrm{B}, \text { rare }} / T_{\mathrm{B}, 32}$ & $\tau_{32}\left(\mathrm{HN}^{13} \mathrm{C}\right)$ & $T_{\mathrm{B}, \text { rare }} / T_{\mathrm{B}, 32}$ & $\overline{\tau_{32}\left(\mathrm{HN}^{13} \mathrm{C}\right)}$ \\
\hline \multirow{3}{*}{$\mathrm{H}^{15} \mathrm{~N}^{13} \mathrm{C} / \mathrm{HN}^{13} \mathrm{C}$} & & \multicolumn{2}{|c|}{ Source size $5^{\prime \prime}$} & \multicolumn{2}{|c|}{ Extended source } \\
\hline & Amb. & $\sim 2.2$ & $\ldots$ & $\sim 0.8$ & $\sim 610$ \\
\hline & Prec. & $\sim 2.2$ & $\ldots$ & $\sim 0.8$ & $\sim 610$ \\
\hline
\end{tabular}

Table 7. $\mathrm{H}_{2}$ densities and molecular column densities of $\mathrm{H}^{13} \mathrm{CO}^{+}$and $\mathrm{HN}^{13} \mathrm{C}$ derived assuming extended emission and a temperature of $21 \mathrm{~K}$ for all molecules, for the precursor component of L1448-mm.

\begin{tabular}{lcc}
\hline \hline & $\mathrm{H}_{2}$ density $\left(\mathrm{cm}^{-3}\right)$ & Column density $\left(\mathrm{cm}^{-2}\right)$ \\
\cline { 2 - 3 } Molecule & \multicolumn{2}{c}{$(0,0)$} \\
\hline $\mathrm{H}^{13} \mathrm{CO}^{+}$ & $5.0 \times 10^{6}$ & $9.3 \times 10^{11}$ \\
$\mathrm{SiO}$ & $1.6 \times 10^{6}$ & $1.2 \times 10^{11}$ \\
$\mathrm{HN}{ }^{13} \mathrm{C}$ & $\lesssim 1.3 \times 10^{6}$ & $3.7 \times 10^{11}$ \\
$\mathrm{H}^{13} \mathrm{CN}$ & $\$ 4.8 \times 10^{6}$ & $3.3 \times 10^{11}$ \\
\hline${ }^{13} \mathrm{CO}^{+}$ & $3.7 \times 10^{7}$ & $2.6 \times 10^{11}$ \\
$\mathrm{SiO}^{13}$ & $3.5 \times 10^{6}$ & $1.4 \times 10^{11}$ \\
$\mathrm{HN}{ }^{13} \mathrm{C}$ & $\$ 7.1 \times 10^{5}$ & $6.8 \times 10^{11}$ \\
$\mathrm{H}^{13} \mathrm{CN}$ & $\$ 3.6 \times 10^{6}$ & $4.2 \times 10^{11}$ \\
\hline
\end{tabular}

et al. 1999), but in this section we also explore the possibility that the gas is at higher temperatures, which is predicted by C-shock models. Finally, we investigate the possibility that the source size is compact.

As the excitation model, we have used the LVG code of JSO6, but using the new collisional coefficients with electrons for $\mathrm{SiO}$ and $\mathrm{HN}^{13} \mathrm{C}$. It should be noted that for any isotopologues, deexcitation rates of the main species were employed, except for $\mathrm{H}^{13} \mathrm{CO}^{+}$. For the latter, a proper calculation was performed and minor differences (less than 5\%) were observed with respect to $\mathrm{HCO}^{+}$rate coefficients. Excitation rates were derived from the detailed balance principle.

\subsubsection{Extended emission with low kinetic temperatures}

The estimated $\mathrm{H}_{2}$ densities and column densities required to produce the observed line intensities are shown in Table 7, assuming that excitation is due to collisions with $\mathrm{H}_{2}$ only. As in JS06, where possible we have used the $J=4 \rightarrow 3$ and $J=3 \rightarrow 2$ lines to derive the $\mathrm{H}_{2}$ densities, as these transitions are more likely to trace the gas processed by the precursor compared to the lower excitation lines. Where this information is not available, we have used the $J=3 \rightarrow 2$ and $J=1 \rightarrow 0$ lines to estimate the $\mathrm{H}_{2}$ densities. The $\mathrm{H}_{2}$ densities for all molecules are a factor of $\sim 10$ times higher than those in Table 3 of JS06. This difference mainly arises because JS06 used main beam temperatures to estimate the $\mathrm{H}_{2}$ densities, which is equal to $T_{\mathrm{B}}$ when the source size exactly fills the beam, whereas in our case we have used $T_{\mathrm{A}}^{*}$, appropriate for extended emission.
We find that an $\mathrm{H}_{2}$ density of $\sim 10^{6} \mathrm{~cm}^{-3}$ can explain the excitation of the neutral species, but the $\mathrm{H}_{2}$ density required for $\mathrm{H}^{13} \mathrm{CO}^{+}$is a factor of $\sim 5$ times larger toward $(0,0)$, and $\sim 10$ times larger toward $(0,-10)$, as previously found by JS06.

If we now assume an $\mathrm{H}_{2}$ density of $1 \times 10^{6} \mathrm{~cm}^{-3}$, fractional ionisations of $5 \times 10^{-4}$ and $7 \times 10^{-3}$ toward $(0,0)$ and $(0,-10)$, repectively, are required to explain the line intensity ratios measured for $\mathrm{H}^{13} \mathrm{CO}^{+}$in the precursor component. These enhanced electron abundances do not make a strong impact on the predicted intensities of $\mathrm{SiO}$ and $\mathrm{HN}^{13} \mathrm{C}$, since they differ by less than 30 per cent for $\mathrm{SiO}$, and by a factor of 2 for $\mathrm{HN}^{13} \mathrm{C}$, with respect to the observed ones. This shows that when using the new collisional coefficients of $\mathrm{SiO}$ and $\mathrm{HN}^{13} \mathrm{C}$ in the calculations, the electron density enhancement in the precursor component is consistent with the measured intensities of both the ion and the neutral species. The LVG model estimates the column densities and opacities of the $\mathrm{H}^{13} \mathrm{CO}^{+} J=3 \rightarrow 2$ lines to be $N=1 \times 10^{12} \mathrm{~cm}^{-2}$ and $\tau_{32}=0.2$ toward $(0,0)$ and $N=3 \times 10^{11} \mathrm{~cm}^{-3}$ and $\tau_{32}=0.05$ toward $(0,-10)$. These opacities are consistent with the observed upper limits in Sect. 4.

\subsubsection{Extended emission with high kinetic temperatures}

Since the collisional coefficients for $\mathrm{H}^{13} \mathrm{CO}^{+}$with electrons used so far in the LVG model are only available for transitions up to $J=5$, which lies at $\sim 62.5 \mathrm{~K}$, for the LVG calculations at higher temperatures we use updated collisional coefficients for $\mathrm{HCO}^{+}$ with electrons (Faure \& Tennyson 2001), with data for transitions up to $J=20$, lying at $\sim 900 \mathrm{~K}$. To ensure that the LVG calculations are converged with respect to the rotational populations, we can only perform LVG calculations for temperatures $\$ 400 \mathrm{~K}$.

If the gas has a kinetic temperature of $100 \mathrm{~K}$, the estimated $\mathrm{H}_{2}$ densities for the neutral species fall to $\sim 3 \times 10^{5} \mathrm{~cm}^{-3}$. For $\mathrm{H}^{13} \mathrm{CO}^{+}$, the estimated $\mathrm{H}_{2}$ densities are $\sim 4 \times 10^{5} \mathrm{~cm}^{-3}$ and $\sim 8 \times$ $10^{5} \mathrm{~cm}^{-3}$ toward $(0,0)$ and $(0,-10)$ respectively. Assuming that the gas density is $3 \times 10^{5} \mathrm{~cm}^{-3}$, the $\mathrm{H}^{13} \mathrm{CO}^{+}$ions would still require fractional ionisations of $3 \times 10^{-4}$ and $8 \times 10^{-4}$ toward $(0,0)$ and $(0,-10)$ respectively to fit the observations.

Performing a similar analysis at $200 \mathrm{~K}, 300 \mathrm{~K}$ and $400 \mathrm{~K}$, we find that toward $(0,0)$, the required $\mathrm{H}_{2}$ density to fit the $\mathrm{H}^{13} \mathrm{CO}^{+}$ data is a factor of 1.5-2 times larger than the $\mathrm{H}_{2}$ density required for the neutral species, and toward $(0,-10)$ this factor is $2.5-4$. The fractional ionisations required to fit the data assuming only one $\mathrm{H}_{2}$ density, range from $3 \times 10^{-4}$ to $2 \times 10^{-3}$ toward $(0,0)$, and from $8 \times 10^{-4}$ to $4 \times 10^{-3}$, toward $(0,-10)$. Without the electron density enhancements, the $\mathrm{H}^{13} \mathrm{CO}^{+} J=3 \rightarrow 2$ lines are 


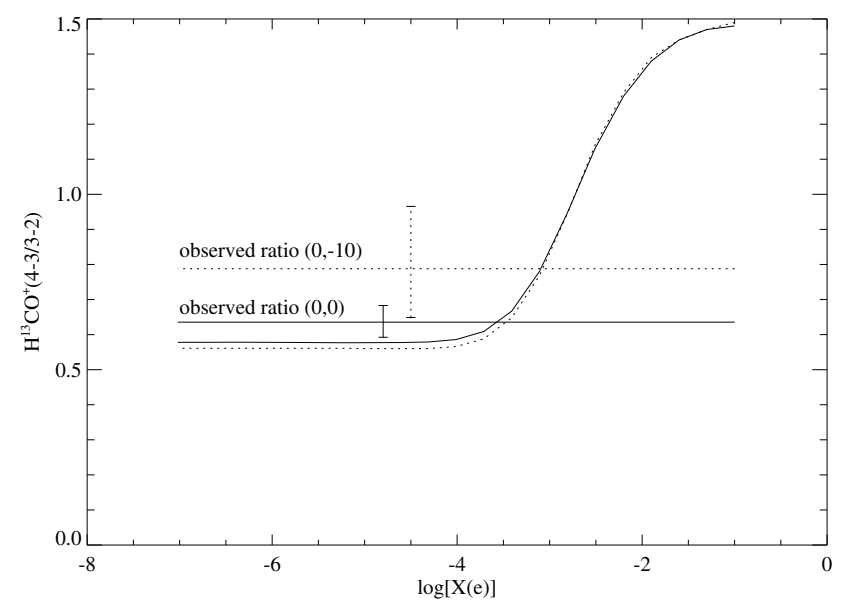

Fig. 3. The $\mathrm{H}^{13} \mathrm{CO}^{+} J=4 \rightarrow 3 / J=3 \rightarrow 2$ ratio as predicted by the LVG model at $100 \mathrm{~K}$ toward $\operatorname{L} 1448-\mathrm{mm}(0,0)$ (solid curve) and L1448-mm $(0,-10)$ (dotted curve), against the electron fractional abundance. The observed $J=4 \rightarrow 3 / J=3 \rightarrow 2$ ratios of the pre-cursor components towards both positions are plotted, and the error bars correspond to the $1 \sigma$ errors in the observations.

underpredicted by $20-60 \%$, and the $\mathrm{H}^{13} \mathrm{CO}^{+} J=4 \rightarrow 3$ lines are underpredicted by $30-70 \%$. The estimated column densities and opacities of $\mathrm{H}^{13} \mathrm{CO}^{+} J=3 \rightarrow 2$ are slightly lower than for the $21 \mathrm{~K}$ case above, with $N=8-9 \times 10^{11} \mathrm{~cm}^{-2}$ and $\tau_{32}=0.2$ toward $(0,0)$ and $N=2 \times 10^{11} \mathrm{~cm}^{-2}$ and $\tau_{32}=0.03$ toward $(0$, -10). Again these opacities are consistent with the upper limits derived from observations (see Sect. 4).

Note that toward $(0,0)$, even though the neutral species require an $\mathrm{H}_{2}$ density of only 1.5-2 times less than that for $\mathrm{H}^{13} \mathrm{CO}^{+}$, the estimated fractional ionisation is very high, up to four orders of magnitude higher than the typical fractional ionisation in dark clouds $\left(\sim 10^{-7}\right)$. This is because the electrons do not significantly affect the excitation of $\mathrm{H}^{13} \mathrm{CO}^{+}$until they reach a fractional abundance of a few $\times 10^{-5}$. This is demonstrated by Fig. 3, where we show the $\mathrm{H}^{13} \mathrm{CO}^{+} J=4 \rightarrow 3 / J=3 \rightarrow 2$ ratio as a function of the electron fractional abundance predicted by the LVG model, for an $\mathrm{H}_{2}$ density of $3 \times 10^{5} \mathrm{~cm}^{-3}$, and a temperature of $100 \mathrm{~K}$. For each position we have used the column densities estimated above, although Fig. 3 shows that variations in the column density have a minimal impact on the predicted $J=4 \rightarrow 3 / J=3 \rightarrow 2$ ratios.

We note that considering the $1 \sigma$ errors in the observations, toward $(0,0)$ the lower limit of the observed $\mathrm{H}^{13} \mathrm{CO}^{+} J=4 \rightarrow$ $3 / J=3 \rightarrow 2$ ratio is approximately equal to that predicted by the LVG model for low electron densities $\left(X(\mathrm{e}) \lesssim 10^{-5}\right)$ for temperatures of $100-400 \mathrm{~K}$. It is therefore possible that there is no need for an electron density enhancement toward $(0,0)$ for these temperatures. However, toward $(0,-10)$, as demonstrated by the error bars plotted in Fig. 3, the need for an electron density enhancement to explain the observations is more compelling; the lower limit of the observed $J=4 \rightarrow 3 / J=3 \rightarrow 2$ ratio is 13-24 per cent larger than that predicted by the LVG model for low electron densities for these high temperatures (compared to within $0.2-12$ per cent toward $(0,0)$ ).

\subsubsection{Compact emission}

Assuming a source size of $5^{\prime \prime}$, converting the $T_{\mathrm{A}}^{*} \mathrm{~s}$ to brightness temperatures, as described in Sect. 4, gives brightness temperatures of $40-50 \mathrm{~K}$ for the $\mathrm{H}^{13} \mathrm{CO}^{+} J=1 \rightarrow 0$ lines, and $15-30 \mathrm{~K}$ for the $\mathrm{HN}^{13} \mathrm{C} J=1 \rightarrow 0$ lines. Therefore, if the emission is compact, the kinetic temperatures of the gas must be $\geq 50 \mathrm{~K}$. The brightness temperatures for the $\mathrm{H}^{13} \mathrm{CO}^{+} J=3 \rightarrow 2$ and $J=4 \rightarrow 3$ lines are only $20-25 \mathrm{~K}$ toward $(0,0)$ and $\sim 7 \mathrm{~K}$ toward $(0,-10)$. For the $\mathrm{HN}^{13} \mathrm{C} J=3 \rightarrow 2$ lines, their brightness temperatures are $\$ 3 \mathrm{~K}$ toward both positions. For gas temperatures $>50 \mathrm{~K}$, our LVG model cannot fit the $\mathrm{HN}^{13} \mathrm{C}$ intensities toward $(0,0)$ and $(0,-10)$, assuming that the molecular excitation is produced by collisions with only $\mathrm{H}_{2}$. This is because we cannot fit the extremely low values of the $J=3 \rightarrow 2 / J=1 \rightarrow 0$ brightness temperature ratios for $\mathrm{HN}^{13} \mathrm{C}$. In fact, the LVG model cannot reproduce the $\mathrm{HN}^{13} \mathrm{C}$ intensities even for kinetic temperatures of $15-30 \mathrm{~K}$. There is a similar problem for $\mathrm{H}^{13} \mathrm{CO}^{+}$; whilst it is possible to find an $\mathrm{H}_{2}$ density and $\mathrm{H}^{13} \mathrm{CO}^{+}$column density to fit the $J=4 \rightarrow 3$ and $J=3 \rightarrow 2$ observations for kinetic temperatures $\geq 50 \mathrm{~K}$, these fits cannot match the extremely high intensities of the $J=1 \rightarrow 0$ lines, and it is impossible to fit the extremely low $J=3 \rightarrow 2 / J=1 \rightarrow 0$ line intensity ratio. Including an electron density enhancement increases the $J=3 \rightarrow 2 / J=1 \rightarrow 0$, making the difference between the model and observed values even larger. Furthermore, observations of $\mathrm{NH}_{3}$ toward L1448-mm indicate that the neutral gas has a temperature of $\sim 20 \mathrm{~K}$ (Curiel et al. 1999), lower than the minimum temperature of $\sim 50 \mathrm{~K}$ required for compact emission. The observational evidence and our modelling results are therefore not consistent with compact emission.

\subsection{Analysis of new positions around the L1448- $\mathrm{mm}$ source}

In this section, we present the analysis of the molecular data toward the newly observed positions L1448-mm $(0,20),(20,0)$ and $(-20,0)$. We use again the LVG approximation as the molecular excitation model. The ion and neutral low- and high$J$ lines of $\mathrm{H}^{13} \mathrm{CO}^{+}$and $\mathrm{HN}^{13} \mathrm{C}$ measured toward these offsets, are needed to constrain not only the spatial extent of the emission arising from the magnetic precursor, but also the extent of the electron density enhancement associated with this early shock stage. As suggested by JS06 for the L1448-mm $(0,0)$ and $(0$, $-10)$ positions, if an electron density enhancement occurs in the precursor, the high- $J$ rotational lines of $\mathrm{H}^{13} \mathrm{CO}^{+}$are expected to be significantly brighter than those of $\mathrm{HN}^{13} \mathrm{C}$.

If the emission is optically thin (see Sect. 4), the line intensity ratio between different transitions is directly related to the excitation temperature of the molecular species. In Table 8 , we show the $J=3 \rightarrow 2 / 1 \rightarrow 0$ integrated intensity ratios for $\mathrm{H}^{13} \mathrm{CO}^{+}$and $\mathrm{HN}^{13} \mathrm{C}$ toward all positions measured in L1448-mm.

For $\mathrm{H}^{13} \mathrm{CO}^{+}$, we find that the $J=3 \rightarrow 2 / 1 \rightarrow 0$ ratio is significantly larger in the precursor component than in the ambient gas (by more than a factor of 3), only toward L1448-mm $(0,0)$ and $(0,-10)$. For $\mathrm{HN}^{13} \mathrm{C}$, this ratio is similar (i.e. differs by less than a factor of 3 ) in both velocity components toward all positions, suggesting that the over-excitation of $\mathrm{H}^{13} \mathrm{CO}^{+}$does not show a large-scale morphology around the L1448-mm core. This clearly contrasts with the idea that the anomalous excitation of $\mathrm{H}^{13} \mathrm{CO}^{+}$is produced by a diffusion/scattering effect (Frerking et al. 1979; Cernicharo et al. 1984).

This can also be qualitatively shown by deriving the $\mathrm{H}_{2}$ densities required to explain the line intensity ratios shown in Table 8, as in Sect. 5.1.1. We assume that the emission is spatially extended and consider a kinetic temperature of $21 \mathrm{~K}$. The estimated $\mathrm{H}_{2}$ densities and molecular column densities are shown in Table 9.

We see that the intensities of the low- and high- $J \mathrm{H}^{13} \mathrm{CO}^{+}$ lines arising from the precursor component toward L1448-mm 
Table 8. $J=3 \rightarrow 2 / 1 \rightarrow 0$ integrated intensity ratios. Data were taken from JS06 and this paper.

\begin{tabular}{|c|c|c|c|c|c|c|}
\hline \multirow[b]{2}{*}{ Molecule } & \multicolumn{2}{|c|}{$(0,20)$} & \multicolumn{2}{|c|}{$(20,0)$} & \multicolumn{2}{|c|}{$(-20,0)$} \\
\hline & Ambient & Precursor & Ambient & Precursor & Ambient & Precursor \\
\hline $\mathrm{H}^{13} \mathrm{CO}^{+}$ & 0.2 & 0.5 & $\lesssim 0.2$ & 0.4 & $\lesssim 0.2$ & 0.09 \\
\hline \multirow[t]{2}{*}{$\mathrm{HN}^{13} \mathrm{C}$} & $\lessgtr 0.1$ & $\lessgtr 0.3$ & $\$ 0.1$ & $\$ 0.1$ & $\lesssim 0.1$ & $\lessgtr 0.3$ \\
\hline & \multicolumn{2}{|c|}{$\overline{(0,0)}$} & \multicolumn{2}{|c|}{$\overline{(0,-10)}$} & \multicolumn{2}{|c|}{$\overline{(0,-20)}$} \\
\hline Molecule & Ambient & Precursor & Ambient & Precursor & Ambient & Precursor \\
\hline $\mathrm{H}^{13} \mathrm{CO}^{+}$ & 0.5 & 1.6 & $\lesssim 0.1$ & 0.6 & $\lesssim 0.5$ & 0.2 \\
\hline $\mathrm{HN}^{13} \mathrm{C}$ & 0.4 & $\lesssim 0.2$ & $\lesssim 0.2$ & $\lesssim 0.1$ & $\lesssim 0.2$ & $\lesssim 0.3$ \\
\hline
\end{tabular}

Table 9. $\mathrm{H}_{2}$ volume densities and molecular column densities of $\mathrm{H}^{13} \mathrm{CO}^{+}$and $\mathrm{HN}^{13} \mathrm{C}$ derived assuming extended emission for both the ambient and the precursor components of L1448-mm.

\begin{tabular}{|c|c|c|c|c|}
\hline \multirow[b]{2}{*}{ Molecule } & \multicolumn{2}{|c|}{$\mathrm{H}_{2}$ density $\left(\mathrm{cm}^{-3}\right)$} & \multicolumn{2}{|c|}{ Column density $\left(\mathrm{cm}^{-2}\right)$} \\
\hline & Ambient & Precursor & Ambient & Precursor \\
\hline \multirow{3}{*}{$\begin{array}{l}\mathrm{H}^{13} \mathrm{CO}^{+} \\
\mathrm{HN}^{13} \mathrm{C}\end{array}$} & & $(0,20)$ & & \\
\hline & $1.4 \times 10^{5}$ & $3.5 \times 10^{5}$ & $4.8 \times 10^{11}$ & $4.0 \times 10^{11}$ \\
\hline & $\lesssim 1.9 \times 10^{5}$ & $\$ 9.2 \times 10^{5}$ & $\lesssim 6.4 \times 10^{11}$ & $\lesssim 1.5 \times 10^{11}$ \\
\hline \multirow{3}{*}{$\begin{array}{l}\mathrm{H}^{13} \mathrm{CO}+ \\
\mathrm{HN}^{13} \mathrm{C}\end{array}$} & & $(20,0)$ & & \\
\hline & $\lesssim 8.8 \times 10^{4}$ & $1.8 \times 10^{5}$ & $\lesssim 5.1 \times 10^{11}$ & $4.3 \times 10^{11}$ \\
\hline & $\lesssim 1.8 \times 10^{5}$ & $\lesssim 3.3 \times 10^{5}$ & $\lesssim 7.2 \times 10^{11}$ & $\lesssim 4.1 \times 10^{11}$ \\
\hline & & $(-20,0)$ & & \\
\hline $\mathrm{H}^{13} \mathrm{CO}^{+}$ & $\lesssim 8.7 \times 10^{4}$ & $7.1 \times 10^{4}$ & $\lesssim 3.5 \times 10^{11}$ & $6.4 \times 10^{11}$ \\
\hline $\mathrm{HN}^{13} \mathrm{C}$ & $1.2 \times 10^{5}$ & $\$ 6.1 \times 10^{5}$ & $1.4 \times 10^{12}$ & $\lesssim 2.0 \times 10^{11}$ \\
\hline
\end{tabular}

$(0,20),(20,0)$ and $(-20,0)$, can be explained by an $\mathrm{H}_{2}$ density that differs only by a factor of 2 with respect to that of the ambient gas, and that is less than the upper limits of the $\mathrm{H}_{2}$ denstities derived from $\mathrm{HN}^{13} \mathrm{C}$ within the precursor component.

These results suggest that the ion $\mathrm{H}^{13} \mathrm{CO}^{+}$fluid does not show any evidence of over-excitation with respect to the neutral fluid toward the newly observed offsets around L1448-mm. This implies that molecular excitation by collisions only with $\mathrm{H}_{2}$, can explain the intensities of the low- and high- $J$ lines of $\mathrm{H}^{13} \mathrm{CO}^{+}$ and $\mathrm{HN}^{13} \mathrm{C}$ measured toward these new positions. The detected over-excitation of the ions toward $\operatorname{L} 1448-\mathrm{mm}(0,0)$ and $(0,-10)$ seems to be restricted to a particular region located in the southern red-shifted lobe of this outflow. This is the expected behavior since very young shocks have been reported by Girart \& Acord (2001) toward these positions.

\section{Discussion and conclusions}

Anomalies in the intensities of the hyperfine components of the strongly polar HCN molecule were reported on large-scales toward the TMC-1 molecular dark cloud by Cernicharo et al. (1984). These authors showed that these anomalies are due to large opacity effects and are produced by the re-emission of radiation coming from the densest part of the core, by a more extended and less dense envelope. If this applies to the L1448-mm case, then we should expect to detect such differences in the excitation of other strongly polar molecular species such as $\mathrm{HCO}^{+}$ and HNC, on large-scales around the L1448-mm core. In Sect. 4, from isotopologue observations we have shown that the precursor component of $\mathrm{H}^{13} \mathrm{CO}^{+}$is optically thin toward $(0,0)$, and if the emission is extended, this component is also optically thin toward $(0,-10)$. This implies that the over-excitation of $\mathrm{H}^{13} \mathrm{CO}^{+}$ in the precursor toward these positions cannot be due to a largescale optical depth/scattering effect.
In view of the recent release of new collisional coefficients of $\mathrm{SiO}$ and HNC with electrons (Faure et al. 2007; Varambhia et al. 2009), we have re-analysed the multi molecular line observations toward L1448-mm $(0,0)$ and $(0,-10)$ of JS06, but taking into account different possibilities for the source size extent and temperature. Assuming extended emission and kinetic temperatures of up to $400 \mathrm{~K}$, and considering that the molecular excitation is due to collisions with only $\mathrm{H}_{2}$, a higher $\mathrm{H}_{2}$ density is required to match the observations of $\mathrm{H}^{13} \mathrm{CO}^{+}$than that needed for the neutral species. For temperatures of 100-400 K, toward L1448- $\mathrm{mm}(0,0)$ it is possible that, considering the $1 \sigma$ errors in the observations, the observations can be explained by a single $\mathrm{H}_{2}$ density for the ions and the neutrals, but toward $(0,-10)$ these errors are not enough to explain the difference in the required $\mathrm{H}_{2}$ densities. This leads to the need for an extra excitation mechanism for $\mathrm{H}^{13} \mathrm{CO}^{+}$in the precursor component of L1448-mm. This extra excitation can be provided by an electron density enhancement of up to a factor of $\sim 10^{4}$. As in JS06, the derived electron density enhancement exceeds those predicted by MHD shock modelling by up to two orders of magnitude (Flower et al. 1996; Flower \& Pineau des Forets 2003). However, simply considering the $1 \sigma$ errors in the observations can reduce the electron density estimates by an order of magnitude. Due to these very large uncertainties, we can conclude that an electron density enhancement can explain the observations, but we cannot quantify it with an accuracy better than one order of magnitude.

If the emission is compact, the gas kinetic temparture must be $\gtrsim 50 \mathrm{~K}$. However, we find that the $\mathrm{HN}^{13} \mathrm{C}$ and $\mathrm{H}^{13} \mathrm{CO}^{+}$lines cannot be fitted in this case, so we conclude that the emitting region associated with the precursor must be $>5^{\prime \prime}$.

The observations toward the L1448-mm $(0,20),(20,0)$ and $(-20,0)$ positions, do not show evidence for an over-excitation of $\mathrm{H}^{13} \mathrm{CO}^{+}$with respect to the neutral $\mathrm{HN}^{13} \mathrm{C}$ molecules in the precursor component of L1448-mm. This suggests that the extramechanism responsible for the over-excitation of the ions has its origin in local phenomena likely linked to the recent interaction of very young C-shocks. Girart \& Acord (2001) have indeed reported very young shocks (with a dynamical age of $\$ 90 \mathrm{yr}$ ) toward the southern position L1448-mm $(0,-10)$, making the probability to detect the magnetic precursor of C-shocks not negligible. Since our results show that the over-excitation of ions is confined to this region, there seems to be strong evidence to suggest that this over-excitation is produced by the electron density enhancement at the magnetic precursor stage of young shocks. Furthermore, the confinement of the over-excitation of the ions also implies that this effect is unlikely to be caused by external UV illumination, as it should be observed tracing the inner regions of the outflow cavity, and on larger spatial scales.

In order to fully understand the extent of the magnetic precursor of L1448-mm, high angular resolution observations of the high- $J$ transitions of $\mathrm{H}^{13} \mathrm{CO}^{+}, \mathrm{HN}^{13} \mathrm{C}$ and $\mathrm{SiO}$ carried out 
with interferometers such as the Submillimeter Array (SMA), are therefore strongly required to measure the source sizes of this molecular emitting regions and to confirm whether or not this emission is optically thin. Observations of very high- $J(J>10)$ transitions are also required to verify whether or not the gas has reached the high temperatures predicted by $\mathrm{C}$-shock models, as these lines should be detected for such temperatures. It is also necessary to search for signs of the electron density enhancement toward other young outflows where narrow $\mathrm{SiO}$ has been detected, such as L1448-IRS3, NGC 1333-IRS4 and NGC 1333-IRS2, in order to investigate whether the electron density enhancement is a common phenomenon in this type of objects, and if it is correlated with the detection of very narrow $\mathrm{SiO}$ emission centred at ambient cloud velocities.

Acknowledgements. This work has been partially supported by the Spanish Ministerio de Ciencia e Innovación under project ESP2007-65812-C0201, and by the Comunidad de Madrid Government under PRICIT project S-0505/ESP-0237 (ASTROCAM).

\section{References}

Bachiller, R., Cernicharo, J., Martín-Pintado, J., Tafalla, M., \& Lazareff, B. 1990, A\&A, 231, 174

Casassus, S., Stahl, O., \& Wilson, T. L. 2005, A\&A, 441, 181

Caselli, P., Hartquist, T. W., \& Havnes, O. 1997, A\&A, 322, 296
Cernicharo, J., Castets, A., Duvert, G., \& Guilloteau, S. 1984, A\&A, 139, L13

Choi, M., Evans, N. J., \& Jaffe, D. T. 1993, ApJ, 417, 624

Curiel, S., Torrelles, J. M., Rodríguez, L. F., Gómez, J. F., \& Anglada, G., 1999, 527,310

Draine, B. T. 1980, ApJ, 241, 1021

Faure, A., \& Tennyson, J. 2001, MNRAS, 325, 443

Faure, A., Varambhia, H. N., Stoecklin, T., \& Tennyson, J. 2007, MNRAS, 382, 840

Flower, D. R., \& Pineau des Forets, G. 2003, MNRAS, 343, 390

Flower, D. R., Pineau des Forets, G., Field, D., \& May, P. W. 1996, MNRAS, 280,447

Frerking, M. A., Wilson, R. W., \& Langer, W. D. 1979, ApJ, 232, 65

Girart, J. M., \& Acord, J. M. P. 2001, ApJ, 552, L63

Jiménez-Serra, I., Martín-Pintado, J., Rodríguez-Franco, A., \& Marcelino, N. 2004, ApJ, 603, L49 (JS04)

Jiménez-Serra, I., Martín-Pintado, J., Viti, S., et al. 2006, ApJ, 650, L135 (JS06) Jiménez-Serra, I., Caselli, P., Martín-Pintado, J., \& Hartquist, T. W. 2008, A\&A, 482,549

Jiménez-Serra, I., Martín-Pintado, J., Caselli, P., Viti, S., \& Rodríguez-Franco, A. 2009, ApJ, 695, 149

Langer, W. D., Wilson, R. W., Henry, P. S., \& Guelin, M. 1978, ApJ, 225, 139

Requena-Torres, M. A., Mercelino, N., Jiménez-Serra, I., et al. 2007, ApJ, 655, 37

Schilke, P., Walmsley, C. M., Pineau des Forets, G., \& Flower, D. R. 1997, A\&A, 321,293

Varambhia, H. N., Gupta, M., Faure, A., Baluja, K. L., \& Tennyson, J. 2009, J. Phys. B: At. Mol. Opt. Phys., 42, 095204

Wilson, T. L. 1999, Rep. Prog. Phys., 62, 143

Ziurys, L. M., Friberg, P., \& Irvine, W. M. 1989, ApJ, 343, 201 